\title{
Time-dependent phase-space characterization of intense charged particle beams
}

\author{
D. Stratakis, ${ }^{*}$ R. A. Kishek, R. B. Fiorito, K. Tian, ${ }^{\dagger}$ I. Haber, P. G. O’Shea, M. Reiser, and J. C. T. Thangaraj \\ Institute for Research in Electronics and Applied Physics, University of Maryland, College Park, Maryland 20742, USA
}

(Received 3 October 2008; published 18 February 2009)

\begin{abstract}
Knowledge of the three-dimensional structure of a charged particle beam bunch is essential for understanding its evolution and for initializing computer simulations, especially when space charge is involved. This paper presents a novel experimental method for time-sliced mapping of the transverse phase space of a space-charge dominated beam based on tomographic principles. The combination of a high precision tomographic diagnostic with fast imaging screens and a gated camera are used to produce phase-space maps of two beams: one with a parabolic current profile and another with a short perturbation atop a rectangular pulse. The correlations between longitudinal and transverse phase spaces are apparent and their impact on the dynamics is discussed.
\end{abstract}

DOI: 10.1103/PhysRevSTAB.12.020101

PACS numbers: 29.27. $-\mathrm{a}, 52.59 . \mathrm{Sa}, 42.30 . \mathrm{Wb}$

\section{INTRODUCTION}

A high brightness and low emittance beam is an a priori requirement for $\mathrm{x}$-ray free electron lasers (FELs) [1], brighter-luminosity high-energy colliders [2], energy recovery linacs [3], and Spallation Neutron Sources [4]. For these applications, longitudinal stability is a fundamental requirement. Density modulations in the injection region, produced, for example, by modulations in the drive laser of a photoinjector or from space charge in the gun [5], can be converted at low energy by space-charge forces into energy modulations [6], which are then frozen-in as the beam gets accelerated into ultrarelativistic velocities [7-9]. These modulations in energy can reappear further downstream in doglegs or compression chicanes [10] and possibly lead to unwanted coherent synchrotron radiation [11]. To mitigate these effects, novel methods have been proposed [7,12] and attempted [13] for creating an ellipsoidal beam bunch in which the line charge density is parabolic. In such beams, the space-charge forces are linear both longitudinally and transversely. Besides the linear spacecharge forces, such a bunch has the advantage of being shape preserving [14]. For proton and ion beams, longitudinal stability is at least as important since the beam spends a longer part of its life in the low-energy region where space-charge forces are strong [15].

It is important to bear in mind that the actual beam distribution is in reality three dimensional, hence any investigation of longitudinal stability needs to account for possible correlations between the transverse and longitudinal dynamics, evidence of which has been reported in previous studies [15-20]. Recent experiments [21] using time-dependent imaging techniques demonstrated, in fact, that the transverse beam distribution is affected by longi-

\footnotetext{
*Present address: Brookhaven National Laboratory, Upton, NY 11973.

${ }^{\dagger}$ Present address: Thomas Jefferson National Accelerator Facility, Newport News, VA 23606.
}

tudinal perturbations and vice versa. However, these previous studies measured the beam only in configuration space, and any knowledge of phase space was inferred from simulations. Characterization of the time-resolved transverse phase space is furthermore important [22], as it allows the tuning and verification of the photoinjector properties. An example of this is emittance compensation $[23,24]$, a process which is used to correct for the correlation between the phase-space angle and the longitudinal position of slices.

A key requirement for addressing such issues, therefore, is the ability to measure the detailed three-dimensional structure of the beam distribution. This information is furthermore crucial for initializing computer simulations. In Ref. [21], for example, the simulations were provided with initial conditions for beam current, mean energy, energy spread, and transverse radius-all as a function of time along the bunch. However, the authors had to make educated guesses about the time variation of the transverse beam slope and emittance, based on time-integrated measurements. Being able to directly measure these quantities will considerably enhance the predictive capability of the simulations.

In this paper we report on a proof-of-principle experiment for demonstrating the possibility of reconstructing the time-resolved phase-space distribution by tomography which provides us with far more information than a timesliced emittance. This work is motivated in large part by the implementation of fast imaging techniques [21] on the long solenoid experiment (LSE), which is a simple scaled setup that uses high-current, low-energy electron beams to experimentally model space-charge effects in higherenergy ion accelerators and intense electron injectors. The tomography algorithm we use is similar to that employed in Refs. [25-28], but has been adjusted to account for the beam space charge [28] by assuming a uniform beam density and hence linear space-charge forces. It is worth noting that the tomographic measurements de- 
scribed herein employ beams that are more intense than those described in any other tomographic measurement.

We report time-resolved phase-space maps for two beams: a long rectangular pulse with a short perturbation in charge density we deliberately introduce, and a parabolic beam bunch that is close to an ellipsoidal distribution. The time resolution in both cases is of the order of a few ns, which is much shorter than the beam bunch length (50$100 \mathrm{~ns}$ ) and therefore sufficient to study the beam dynamics for our beam conditions. We show that time-resolved tomography can provide entirely different phase-space maps than the integrated version of the diagnostic. The method we have developed can produce time-resolved phase-space maps useful for initializing simulation codes and optimizing the injection and transport of high-quality beams downstream for future accelerator applications. We emphasize that this work describes and demonstrates a new methodology which can be applicable to any beam pulse using imaging methods with the appropriate time resolution for the pulse duration.

The outline of this paper is as follows: In Sec. II we review the tomographic algorithm. In Sec. III we describe our experimental configuration. In Sec. IV we describe the experimental results. Finally, we present our conclusions in Sec. V.

\section{PHASE-SPACE TOMOGRAPHY}

Tomography is a methodology whose goal is the reconstruction of a higher $N$-dimensional space from projections at a lower $(N-1)$ dimension, e.g., reconstruction of a $2 \mathrm{D}$ image from 1D profiles obtained at various angles. The central methodology is based on the theorem due to Radon [29].

Suppose that $f(x, y)$ corresponds to the two-dimensional distribution that we are interested to reconstruct. Let $\rho=$ $x \cos \theta+y \sin \theta(0 \leq \theta \leq \pi)$ be the equation of a line $L$ that is at a distance $\rho$ from the origin and at an angle, $\theta$, from the $y$ axis. The line integral along $L$ is defined as [30]

$$
\begin{aligned}
\hat{f}_{\theta}(\rho) & =\int_{L} f(x, y) d s \\
& =\int_{-\infty}^{\infty} \int_{-\infty}^{\infty} d x d y f(x, y) \delta(\rho-x \cos \theta-y \sin \theta) .
\end{aligned}
$$

$\hat{f}_{\theta}(\rho)$ is known as the Radon transform of the distribution $f(x, y)$. A projection of $f(x, y)$ is formed if we combine a set of line integrals. The simplest projection is a collection of "parallel" line integrals as is given by Eq. (1) for a fixed $\theta$ while $\rho$ is varied. Then, several algorithms are available to recover the original distribution from such projection data, e.g., algebraic reconstruction technique, maximum entropy tomography, filtered-backprojection algorithm (FBA), etc. The FBA algorithm is the most common method to reconstruct a two-dimensional image and is the algorithm that we use and hence we describe along the lines below. If $F(u, v)$ is the two-dimensional Fourier transform of the function $f(x, y)$, then its inverse Fourier transform is given by

$$
f(x, y)=\int_{-\infty}^{\infty} \int_{-\infty}^{\infty} F(u, v) e^{j 2 \pi(u x+v y)} d u d v .
$$

By exchanging the rectangular coordinate system in the frequency domain $(u, v)$ for a polar coordinate system $(w, \theta)$, Eq. (2) becomes

$$
f(x, y)=\int_{0}^{\pi} \int_{-\infty}^{\infty} F(w, \theta)|w| e^{j 2 \pi w \rho} d w d \theta .
$$

Using the Fourier slice theorem [30], we can substitute the one-dimensional Fourier transform of the Radon transform at angle, $\theta, S_{\theta}(w)=\int_{-\infty}^{\infty} \hat{f}_{\theta}(\rho) e^{-j 2 \pi w \rho} d \rho$, for the twodimensional Fourier transform $F(w, \theta)$. Then Eq. (3) becomes

$$
f(x, y)=\int_{0}^{\pi} \int_{-\infty}^{\infty} S_{\theta}(w)|w| e^{j 2 \pi w \rho} d w d \theta .
$$

Furthermore, Eq. (4) can be simplified to

$$
f(x, y)=\int_{0}^{\pi} Q_{\theta}(\rho) d \theta,
$$

where $Q$ is given by $Q_{\theta}(\rho)=\int_{-\infty}^{\infty} S_{\theta}(w)|w| e^{j 2 \pi w \rho} d w$ and often is called "filtered projection" [30]. Therefore, if a number of parallel projections corresponding to angles from 0 to $\pi$ are known, the tomographic image reconstruction can be done by two steps: first, the filtered projections $Q$ are obtained and, second, the resulting projections for each angle are added [using Eq. (5)] to form an estimate of the distribution $f(x, y)$. The accuracy of the reconstruction will depend on the number of projections and the corresponding angular resolution between them.

Next we will relate the Radon transform to the beam phase-space mapping. Suppose that $\mu\left(x_{0}, x_{0}^{\prime}\right)_{z_{0}}$ is the phase-space distribution at a certain location $z_{0}$ in the beam line. If $\mu\left(x, x^{\prime}\right)_{z_{1}}$ is the phase distribution at another location $z_{1}$ then by assuming that we have a linear system, the particle motion at the two positions obeys

$$
\left(\begin{array}{c}
x \\
x^{\prime}
\end{array}\right)=M\left(\begin{array}{c}
x_{0} \\
x_{0}^{\prime}
\end{array}\right)
$$

where

$$
M=\left(\begin{array}{ll}
M_{11} & M_{12} \\
M_{21} & M_{22}
\end{array}\right)
$$

is the transport matrix between $z_{0}$ and $z_{1}$. In the analysis we will assume that between those two points at least one magnet (quadrupole or solenoid) is present. Placing a screen along the beam path at $z_{1}$ provides a twodimensional projection of the four-dimensional phasespace distribution $f\left(x, x^{\prime}, y, y^{\prime}\right)_{z_{1}}$ which is given by 


$$
g(x, y)_{z_{1}}=\iint f\left(x, x^{\prime}, y, y^{\prime}\right)_{z_{1}} d x^{\prime} d y^{\prime}
$$

Integration of this distribution over $y$ leads to the spatial beam profiles along $x$ :

$$
c(x)_{z_{1}}=\int g(x, y)_{z_{1}} d y=\int\left(\iint f\left(x, x^{\prime}, y, y^{\prime}\right)_{z_{1}} d y d y^{\prime}\right) d x^{\prime} .
$$

In Ref. [28] it was shown that a simple scaling equation relates those beam profiles to the Radon transform, $\hat{\mu}_{\theta, z_{0}}(\rho)$ of the transverse phase space $\mu\left(x_{0}, x_{0}^{\prime}\right)_{z_{0}}$. That is

$$
\hat{\mu}_{\theta, z_{0}}(\rho)=\hat{\mu}_{\theta, z_{0}}(x / s)=s c(x)_{\theta, z_{1}},
$$

where $s$ is the scaling factor given by $[25,28] s=$ $\sqrt{M_{11}^{2}+M_{12}^{2}}$ and $\theta$ is the corresponding projection angle, given by $[25,28] \tan \theta=M_{12} / M_{11}$. Therefore, by applying Eqs. (1)-(5) and (9), tomographic techniques can recover the transverse phase-space distribution of a beam using its projections in configuration space. Those projections can be obtained by means of varying the strength of the magnet upstream of the imaging screen (and therefore modifying the transport matrix $M$ ) which has the effect of rotating the distribution in phase space through a given angle, $\theta$.

The technique is similar to a quad scan except that the entire profile on a screen is collected for each magnetic field strength, instead of simply a beam size. In contrast to standard techniques, such as a double-slit phase-space measurement, tomography does not occupy a long section of beam pipe nor does it require additional hardware beyond an imaging diagnostic downstream of one or more magnets that can be scanned.

Tomographic techniques typically involve quadrupoles to rotate the phase-space distribution $[25,28]$. However, recently we have extended the standard tomographic phase-space retrieval method to use solenoids [31], which is advantageous for characterizing low-energy transport systems and photoinjectors. Our reconstruction algorithm successfully incorporated the effects of linear space-charge forces and the resolution of the reconstructed transverse phase space was extensively studied; it was concluded that 48 projections in total were required with solenoid fields selected such that the angular resolution is less than $14^{\circ}$, to achieve a sufficiently high resolution phase-space map. A comparison of the tomographic reconstructed phase space with simulation code results showed good agreement for an emittance and space-charge dominated beam. To quantify this agreement, the values of the $4 \times \mathrm{rms}$ emittances for both beams were calculated from their phase spaces. It was found that for a beam without space charge the agreement is superior and in the presence of space charge there is a maximum $10 \%$ error in the emittance calculated by tomography [31].
In our present experiment, we employ the same approach described in Ref. [31] to recover transverse beam phase space. The only difference is the combined use of fast phosphor screens and a gated camera (described in the next section) to map the phase space in narrow (3-10 ns) longitudinal (time) slices within the beam pulse. We note that, for faster time resolution, optical transition radiation (OTR) or Cherenkov generating screens can be employed for imaging by using sub-ns gated or streak cameras. We will present 3 ns time-resolved phase-space maps here to illustrate the method, since this resolution is sufficient to show dynamical changes in the beam profile and transverse phase space for our beam conditions and well illustrates the method.

\section{EXPERIMENTAL SETUP}

The experiment was carried out on the long solenoid experiment (LSE) at the University of Maryland [21] which is illustrated in Fig. 1. Our transport line consists of a thermionic electron gun, two short solenoids, S1 and S2, and a phosphor screen located immediately downstream of the solenoids. The electron gun is a variableperveance gridded gun which can produce a wide range of beam parameters and its energy varies from 1-5 keV. Parameters that used for our experiment as well design details about the LSE configuration are demonstrated in Table I. The cathode has a Pierce geometry and a planar configuration consisting of the heater, cathode, and grid. The radius of the cathode is $4 \mathrm{~mm}$ and a $3.2 \mathrm{~mm}$ diameter aperture (at $z=0 \mathrm{~cm}$ ), $22 \mathrm{~mm}$ downstream from the cathode is used to control the beam current that is measured by

(a)
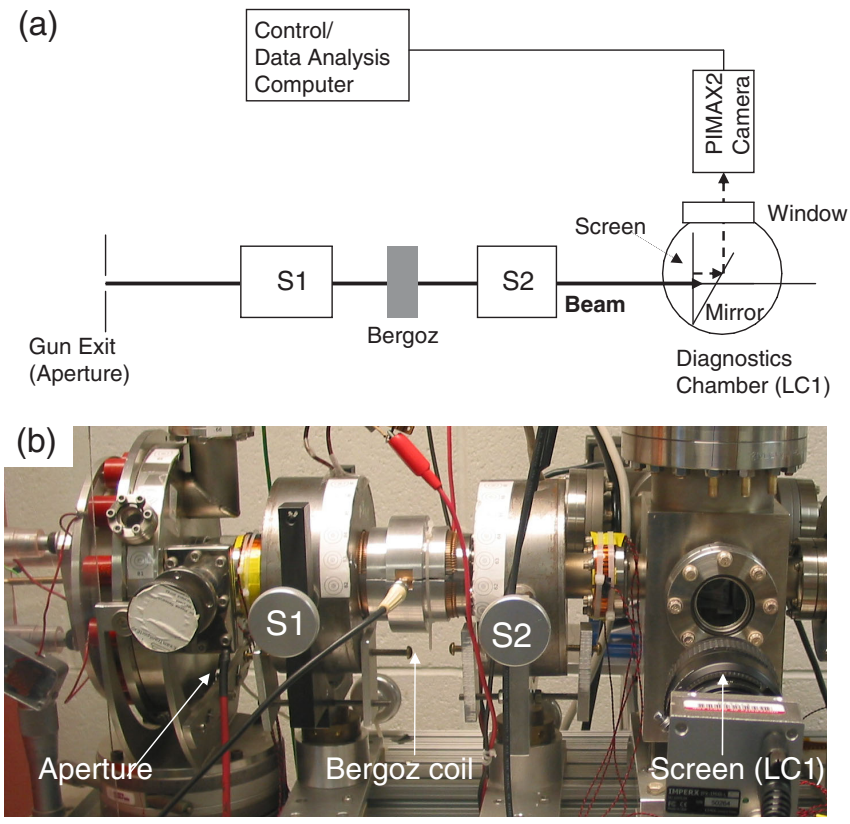

FIG. 1. (Color) Experimental configuration of the LSE: (a) Schematic layout; (b) actual configuration. 
TABLE I. Beam parameters and LSE design specifications.

\begin{tabular}{ll}
\hline \hline Experimental details & \\
\hline Beam energy & $5 \mathrm{keV}$ \\
Location of S1 & $13.1 \mathrm{~cm}$ \\
Location of S2 & $29.5 \mathrm{~cm}$ \\
Effective length of S1 & $4.34 \mathrm{~cm}$ \\
Effective length of S2 & $4.24 \mathrm{~cm}$ \\
Magnetic field of S1 & $97.8 \mathrm{G}$ \\
Magnetic field of S2 & $60.0 \mathrm{G}$ \\
Location of Bergoz FCT & $18.5 \mathrm{~cm}$ \\
Location of the screen (LC1) & $43.0 \mathrm{~cm}$ \\
\hline \hline
\end{tabular}

a Bergoz fast current transformer (FCT). The beam size is controlled by the two solenoids, S1 and S2. As described in Ref. [31], for our tomographic reconstruction we varied the magnetic field strengths of those two solenoids incrementally and obtained a beam image for each setting at a phosphor screen location (at a diagnostic chamber we call LC1). Then, the spatial beam profiles were obtained using Eq. (8) and related to phase-space projections by Eq. (9). By adjusting the transfer matrices [28] in the algorithm, the phase-space maps can be constructed for either the initial beam distribution at the aperture or for the location at the screen. In this paper we show both results. The phase spaces shown in this study are recovered for the default solenoid strength settings illustrated in Table I.

In order to achieve time-dependent phase-space maps within our beam pulse, we employ imaging screens consisting of a fast $\mathrm{ZnO}$ :Ga phosphor deposited on a quartz plate produced by Lexel Imaging Systems, Inc. The phosphor is inorganic and emits in the near UV with peak close to $390 \mathrm{~nm}$ (equivalent to $3.2 \mathrm{eV}$ ) and the decay time is 2.4 ns. Previous experiments [21] demonstrated that the fast phosphor has a linear response to the charge impinging on it. A variable gate, intensified charged-coupled device (ICCD) camera (PIMAX2; Princeton Instruments Inc.) was used to capture the light generated from the screen within a gate time as narrow as 3 ns. The PIMAX2 camera has an image resolution of $512 \times 512$ pixels and a 16-bit dynamic range, which provides a very wide intensity range for detecting even weak signals and halos.

To investigate the evolution of longitudinal perturbations we intentionally produced density modulations close to the beam source by modifying the electronics $[6,32]$ of our gridded electron gun [16]. In this type of gun, a grid $0.15 \mathrm{~mm}$ away from the cathode acts as a gate. Ordinarily it is biased negatively relative to the cathode so as to suppress electron emission. Periodically at $60 \mathrm{~Hz}$, a positive pulse (of $80 \mathrm{~V}$ amplitude) is applied to the grid to extract the beam. Typically, we use a bias voltage of $35 \mathrm{~V}$, which means the grid-cathode potential is of the order of $80-$ $35=45 \mathrm{~V}$ and the gun operates in space-charge-limited saturation mode. By increasing the negative bias voltage to $55 \mathrm{~V}$, the grid-cathode potential difference is reduced and the gun operates in triode amplification mode, in which any perturbation to the grid voltage waveform is translated into a perturbation to the beam current. The grid-cathode pulse voltage is generated by a pulser which is composed of an external triggering circuit, a pulse forming line (PFL), a 2N3507 transistor, and a dc charging power supply. The longitudinal perturbation is created by connecting a cable at the middle of the PFL through a "T" connector. When the gun is working in amplification mode, small changes in the bias voltage produce large changes in the output current. The parabolic beam is produced by applying a lowpass filter to the rectangular, unperturbed grid pulse. A more detailed description about the generation of those perturbations can be found in Refs. [6,32].

\section{EXPERIMENTAL RESULTS AND DISCUSSION}

In this section we first discuss the results with the rectangular pulse, and then with the parabolic pulse.

\section{A. Rectangular pulse with perturbation}

For this experiment we set the bias voltage to $55 \mathrm{~V}$ so as to operate in the triode amplification mode. Note that, although we are increasing the density of electrons within the perturbation, our use of an aperture combined with the beam dynamics inside the $\mathrm{A} / \mathrm{K}$ gap result in a reduction of beam current downstream due to scraping at the aperture [16]. The current profiles, measured at the Bergoz FCT, with and without perturbation are illustrated in Fig. 2. For the experiment, the measured current without perturbation of our $60 \mathrm{~Hz}, 5 \mathrm{keV}$ beam was $25.0 \mathrm{~mA}$ and the pulse length was $100 \mathrm{~ns}$. The width of the perturbation was about



FIG. 2. (Color) Beam signal at the Bergoz showing the rectangular beam with a negative perturbation. The red/black traces show the beam current with perturbation on/off. The perturbation aptitude is roughly $25 \%$ of that of the main beam. The camera gates used were $10 \mathrm{~ns}$ (solid line) and $100 \mathrm{~ns}$ (dotted line). 
$7 \mathrm{~ns}$, and its amplitude corresponding to about a $25 \%$ reduction in beam current. We note that, due to the fact that the perturbation is relatively long with respect to the transverse dimension, the transverse forces experienced by each slice of the beam are not expected to be affected significantly by longitudinal variations in the beam properties.

In order to cover the whole perturbation range and obtain time-resolved images of the charge particle beam on the screen, a 10 ns wide gate is applied to the ICCD camera (along the solid line in Fig. 2). Each photo is the result of 50 frame integrations. As described in Sec. II, to reconstruct the phase space, 48 beam photos at LC1 for different solenoids settings where collected. Then, the same experiment is repeated but with the perturbation turned off.

Figure 3 shows the tomographically reconstructed phase space at the location of the aperture right at the gun exit $(z=0 \mathrm{~cm})$. To reveal more details about the phase-space distribution, we plot $x^{\prime}-x \phi$ versus $x$, where $\phi$ is the phase-space slope defined by [22]

$$
\phi=-\frac{\left\langle x x^{\prime}\right\rangle}{\left\langle x^{2}\right\rangle}
$$

and $\left\langle x^{2}\right\rangle$ and $\left\langle x x^{\prime}\right\rangle$ are the second moments of the beam distribution that are obtained from the measured phase space. From the phase space, we can also extract the $2 \times$
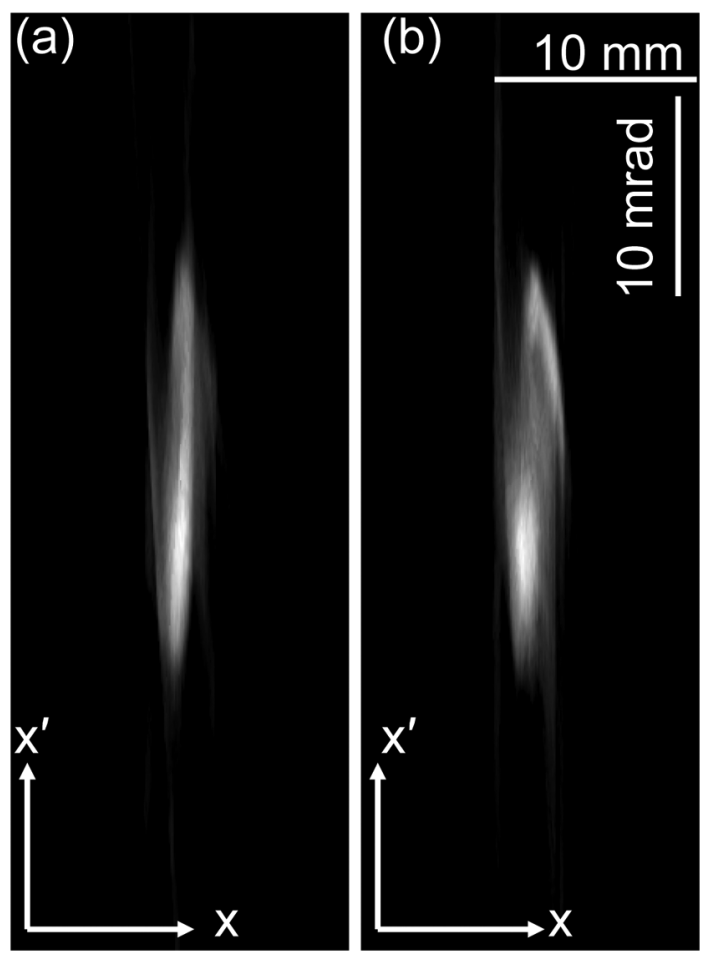

FIG. 3. Beam phase-space distribution in $x$ - $x^{\prime}$ within a $10 \mathrm{~ns}$ gate centered around the perturbation, recovered by tomography and backprojected to the gun aperture $(z=0 \mathrm{~cm})$. (a) Without, and (b) with the perturbation. The subtracted slope $\phi$ is shown in Table II.
TABLE II. Beam parameters for the rectangular beam, measured from phase-space tomography at the aperture plane. Note that $X_{0}$ is the $2 \times r m s$ beam radius, $X_{0}^{\prime}$ is the slope of the beam envelope [Eq. (11)], $\phi_{0}$ is the phase-space slope [Eq. (10)], and $\varepsilon_{0}$ is the $4 \times \mathrm{rms}$ emittance. The subscript " 0 " indicates that those measurements are conducted at $z=0 \mathrm{~cm}$.

\begin{tabular}{lcccc}
\hline \hline & $\begin{array}{c}X_{0} \\
(\mathrm{~mm})\end{array}$ & $\begin{array}{c}X_{0}^{\prime} \\
(\mathrm{mrad})\end{array}$ & $\begin{array}{c}\phi_{0} \\
(\mathrm{mrad} / \mathrm{mm})\end{array}$ & $\begin{array}{c}\varepsilon_{0} \\
(\mu \mathrm{m})\end{array}$ \\
\hline Perturbation off & $1.6 \pm 0.2$ & $17 \pm 2$ & $6.5 \pm 0.7$ & $26 \pm 3$ \\
Perturbation on & $1.6 \pm 0.2$ & $21 \pm 2$ & $9.7 \pm 1.0$ & $30 \pm 3$ \\
\hline \hline
\end{tabular}

rms beam radius, $X$, the $4 \times r m s$ emittance, $\varepsilon$, and the slope of the beam envelope, $X^{\prime}$, defined as

$$
X^{\prime}=\frac{d X}{d z}=\frac{2\left\langle x x^{\prime}\right\rangle}{\sqrt{\left\langle x^{2}\right\rangle}}
$$

Measurement of these parameters can be useful in correctly initializing simulation codes.

Table II indicates the initial measured values of $\phi$ and $X^{\prime}$ at $z=0$ obtained from the phase spaces in Fig. 3. It suggests that the initial beam distribution is considerably affected by the longitudinal perturbation. Specifically, the measured phase-space slope, $\phi$, at the aperture is higher when the perturbation is on (relative to the unperturbed case). This result is consistent with previous experimental measurements [21] showing that the perturbed beam expands faster transversely in the gun, resulting in a larger beam slope at the gun exit. Furthermore, the beam emittance is also higher in the perturbed beam. Note, however, that the two emittances lie within the possible measurement error of tomography, which is $10 \%$, as described previously.

Figure 4 shows images of the same distributions depicted in Fig. 3, but reconstructed at a plane $43 \mathrm{~cm}$ downstream (at the screen position in LC1). Table III illustrates the corresponding beam parameters. The first row in Fig. 4 indicates the beam image on the screen (LC1) and the second shows the corresponding tomographically reconstructed phase spaces at that location when the perturbation is off (left column) and on (right column). Interestingly, the distributions look quite different. In configuration space the beam sizes are not equal and the beam with perturbation appears smaller, possibly due to the different spacecharge forces it experiences relative to the main beam as a result of the difference in current. Note also, the different structure in the phase space of the two distributions, indicating hollowness when the perturbation is on that is not seen otherwise. Such a density perturbation after interaction with the external transport and accelerator environment can excite transverse space-charge waves [6].

A similar structure within the measured distributions like that shown in Figs. 3 and 4 has also been observed in Refs. [21,31]. Particle-in-cell simulations on Ref. [31] revealed that such hollowness in the beam distribution 

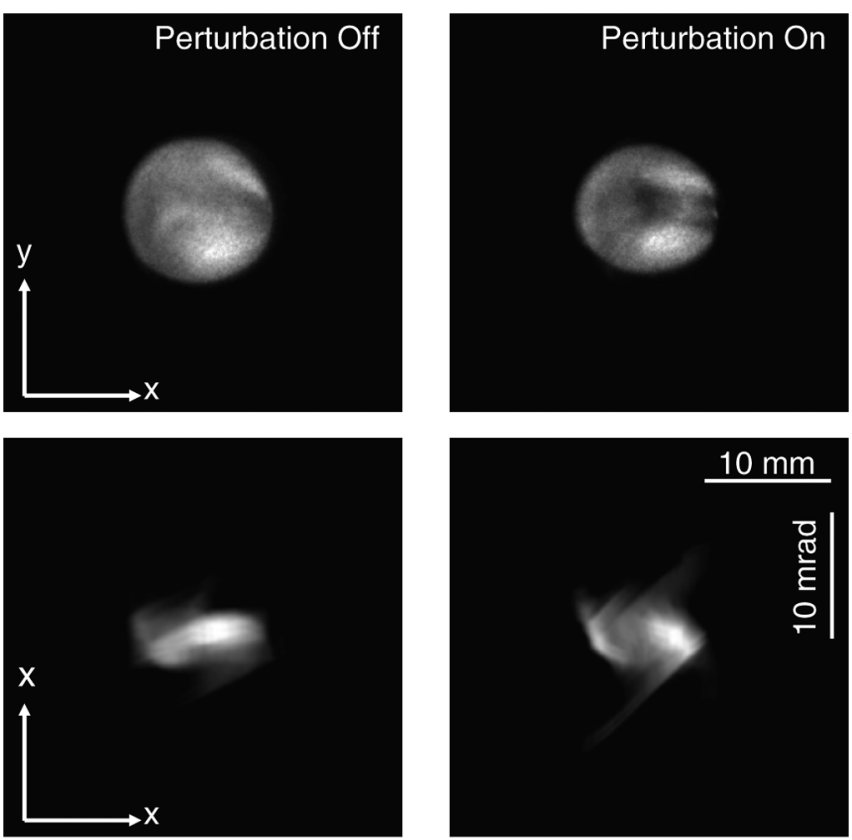

(a)

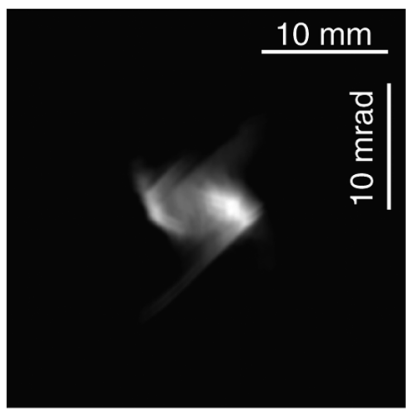

(b)
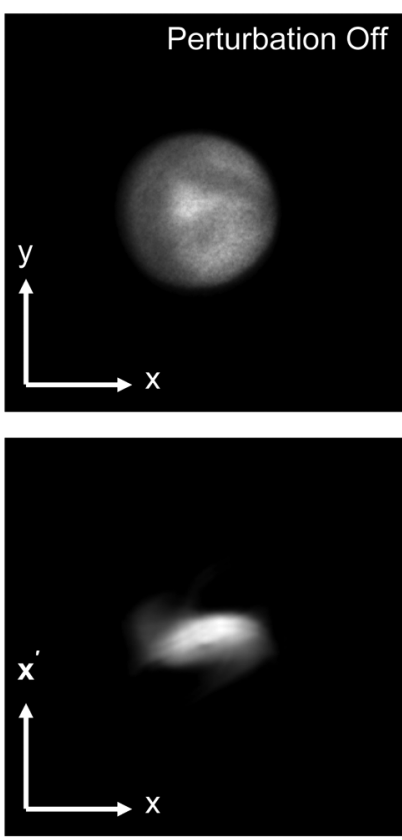

(a)
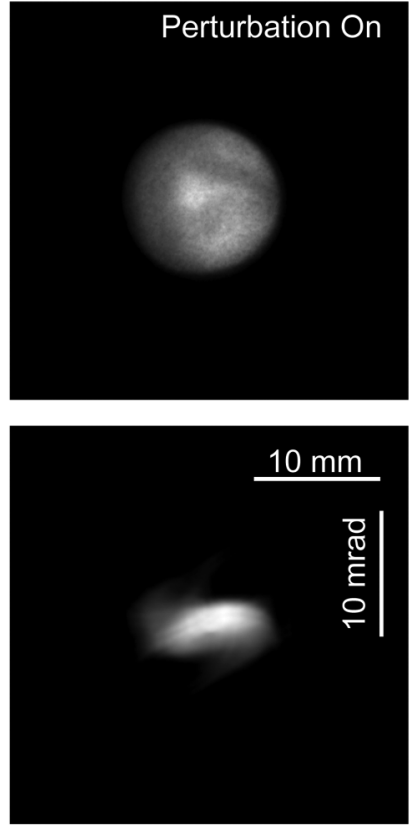

(b)

FIG. 4. Beam configuration space (top) and measured $x-x^{\prime}$ phase space by tomography (bottom) reconstructed at LC1: (a) Without, and (b) with the perturbation. The subtracted slope $\phi$ is shown in Table III.

most likely results partly from the nonuniform distribution that beam possesses when it emerges from the gun cathode, and partly from the aperture used to collimate the beam (see Fig. 1). Note that simulations were initialized right at the gun exit; however, we believe that a detailed analysis of the problem requires simulations that include the electron gun, a task that extends beyond the scope of the present work.

The higher emittance measured for the perturbed case could be due to the transverse mismatch caused by the different initial conditions within the perturbation. A second reason could be the gate window (10 ns) which is larger than the width of the perturbation. Hence, the actual phase space varies on a shorter time scale than the image integration time. The experiments reported here were a first test and proof-of-principle of time-sliced tomography. Future experiments with a smaller gate width should be

TABLE III. Beam parameters for the rectangular beam, measured from phase-space tomography at LC1. The subscript $s$ indicates that those measurements are conducted at the screen in LC1 $(z=43 \mathrm{~cm})$.

\begin{tabular}{lcccc}
\hline \hline & $\begin{array}{c}X_{s} \\
(\mathrm{~mm})\end{array}$ & $\begin{array}{c}X_{s}^{\prime} \\
(\mathrm{mrad})\end{array}$ & $\begin{array}{c}\phi_{s} \\
(\mathrm{mrad} / \mathrm{mm})\end{array}$ & $\begin{array}{c}\varepsilon_{s} \\
(\mu \mathrm{m})\end{array}$ \\
\hline Perturbation off & $5.7 \pm 0.5$ & $18 \pm 2$ & $7.4 \pm 0.7$ & $28 \pm 3$ \\
Perturbation on & $5.3 \pm 0.5$ & $17 \pm 2$ & $6.7 \pm 1.0$ & $37 \pm 4$ \\
\hline \hline
\end{tabular}

FIG. 5. Beam configuration space (top) and measured $x$ - $x^{\prime}$ phase space by tomography (bottom) reconstructed at the screen location: (a) Without, and (b) with the perturbation. The subtracted phase-space slopes, $\phi$, are $7.4 \mathrm{mrad} / \mathrm{mm}$ in both cases. Images are obtained within a 100 ns gate encompassing the entire beam.

able to address this issue in more detail and resolve the relative contributions of the two causes.

To compare the time-sliced tomography results to ordinary, integrated, tomography we repeat the experiment described above but we increase the gate width to $100 \mathrm{~ns}$ so as to encompass the entire beam pulse (the dotted line in Fig. 2).

Figure 5 illustrates the beam distribution in configuration space and phase space without perturbation [Fig. 5(a)] and with perturbation [Fig. 5(b)] at LC1. We cannot distinguish between the two cases; either in configuration space or in phase space. To quantify any differences, we calculated the beam emittances and found that they differ by less than $1 \%$. Hence, in contrast to time-resolved measurements, time-integrated measurements did not reveal any significant differences in the transverse beam distribution when a beam was propagating with and without longitudinal perturbation. This observation highlights the necessity of using appropriately fast diagnostics for studying detailed phase space in rapidly varying beams.

\section{B. Parabolic beam pulse}

We now turn to the parabolic beam, whose physics is of interest to the generation and transport of ellipsoidal bunches as stated earlier. Following the discussion in Sec. II, we produced a parabolic beam current profile by applying the low-pass filter to the grid pulse. Our aim is to 


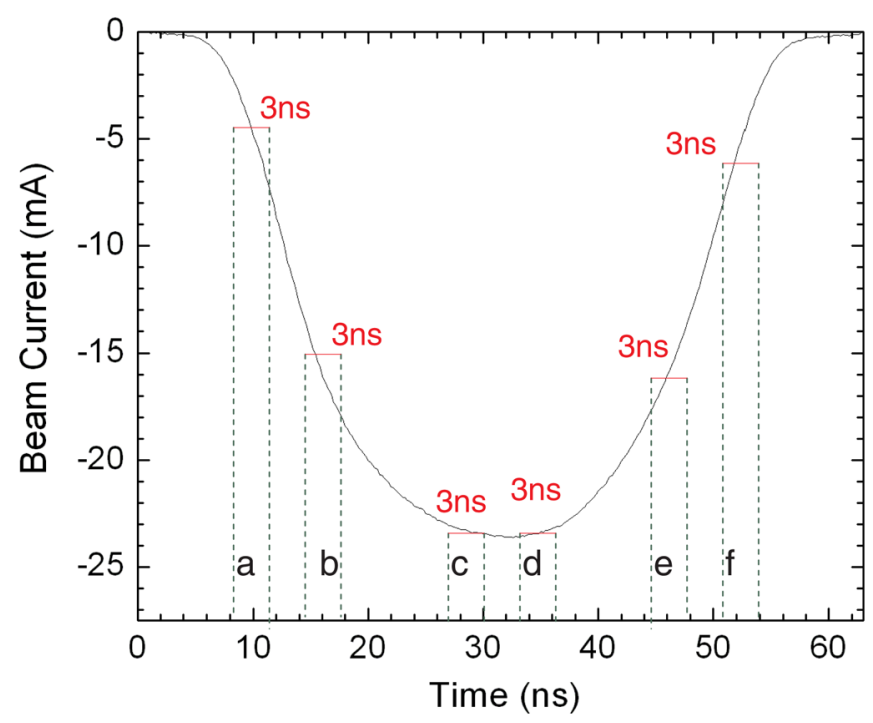

FIG. 6. (Color) Signal collected at the Bergoz coil showing the longitudinal current profile of the parabolic beam, and indicating the approximate locations of the camera gates.

map transverse phase space along several slices within this pulse and study the correlations between longitudinal and transverse dynamics.

For the experiment, the measured peak current of our $60 \mathrm{~Hz}, 5 \mathrm{keV}$ beam was $23.5 \mathrm{~mA}$, the pulse length was $60 \mathrm{~ns}$, and the bias voltage was set at $60 \mathrm{~V}$. Figure 6 shows the longitudinal current profile from the signal at the Bergoz FCT, as well as the position of the slices used in our phase-space measurement (labeled as a, b, c, d, e, and $\mathrm{f}$ ).

By setting the ICCD camera gate window at $3 \mathrm{~ns}$, and moving it progressively from the beam head toward the tail we can collect a number of beam images at the screen (LC1), each corresponding to a 3-ns beam slice. Fig- ure 7 (top) shows the resulting beam distribution in configuration space for the six different slices (a, b, c, d, e, and f). Each photo is the result of 55 frame integrations. Examination of Fig. 7 indicates that the slices vary in size. To illustrate this point we plotted the measured size versus time in Fig. 8(a). The dashed line is the corresponding measured beam size at the beam aperture. Note that all slices at that point have radius equal to $1.6 \mathrm{~mm}$, which is in agreement with aperture radius. As the beam propagates further away, because each slice has a different current, it will evolve differently in the transverse direction. Thus, in $\mathrm{LC} 1$ there will be a variation of beam size within the pulse, and this is clearly depicted by the solid line in Fig. 8(a).

Following the procedure discussed in Sec. II, we scan the solenoids (through a span of $177^{\circ}$ in phase space) to collect 48 beam photos from the screen and reconstruct the phase space for each of the six slices. Figure 7 shows the measured phase space by tomography in LC1. Both configuration space images and the phase-space distributions reveal a detailed structure that differs from slice to slice. As discussed previously, part of the structure inside the phase space arises from the nonuniform distribution at the gun cathode. As pointed out in Ref. [31], such structure scales with the beam intensity. Therefore, since each slice of the parabolic bunch has a different current, the space-charge forces experienced by each slice differ, and henceforth the final structure of the phase space differs as well.

Furthermore, like the configuration space images, the phase spaces depend on the position along the beam; both exhibit symmetry about the peak of the pulse. Figure 8(b) shows the measured beam emittance at LC1 as calculated from those phase spaces (solid line). The dashed line indicates the emittance measured at the aperture also by tomography. Given that the tomography measured error is about $10 \%$, our results do not reveal any significant emittance difference between the aperture and LC1. However,

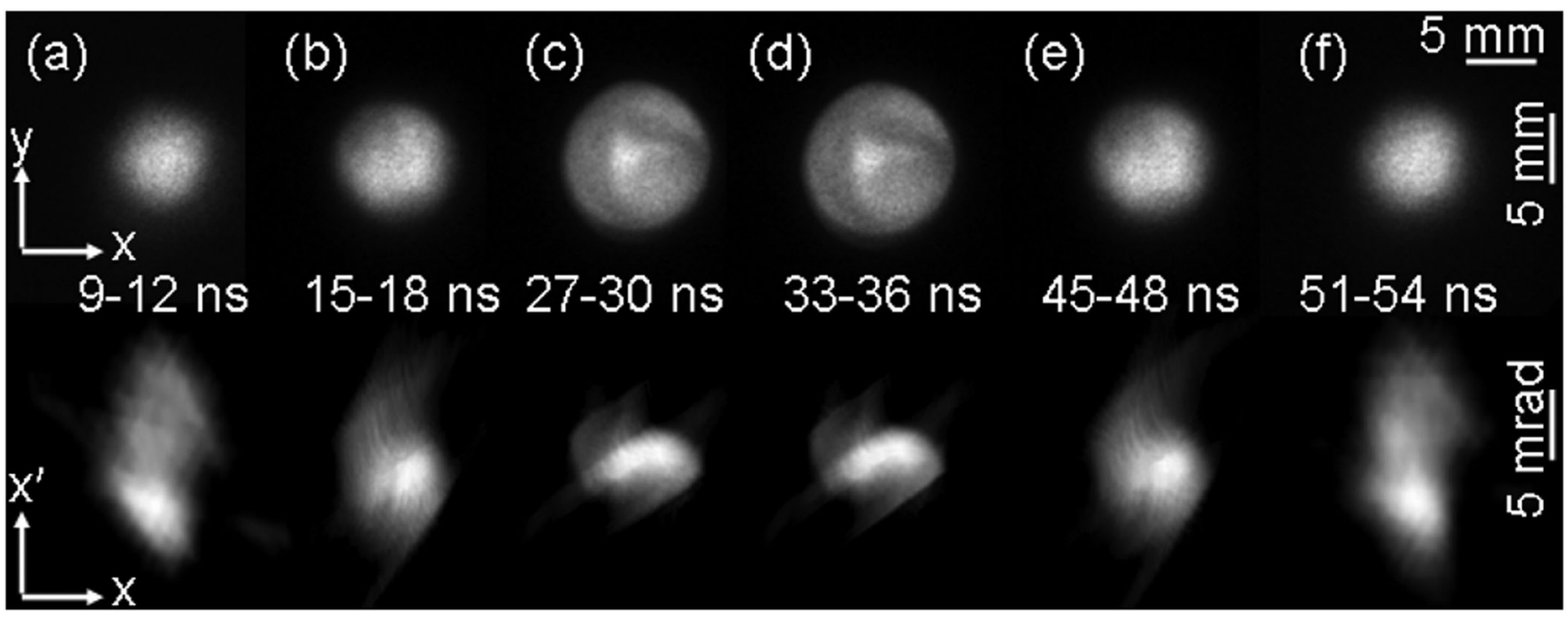

FIG. 7. Beam in configuration space (top) and in $x$ - $x^{\prime}$ phase space (bottom) for slices a, b, c, d, e, and f, reconstructed at LC1. Phase spaces are generated using the initial conditions shown in Fig. 8. 

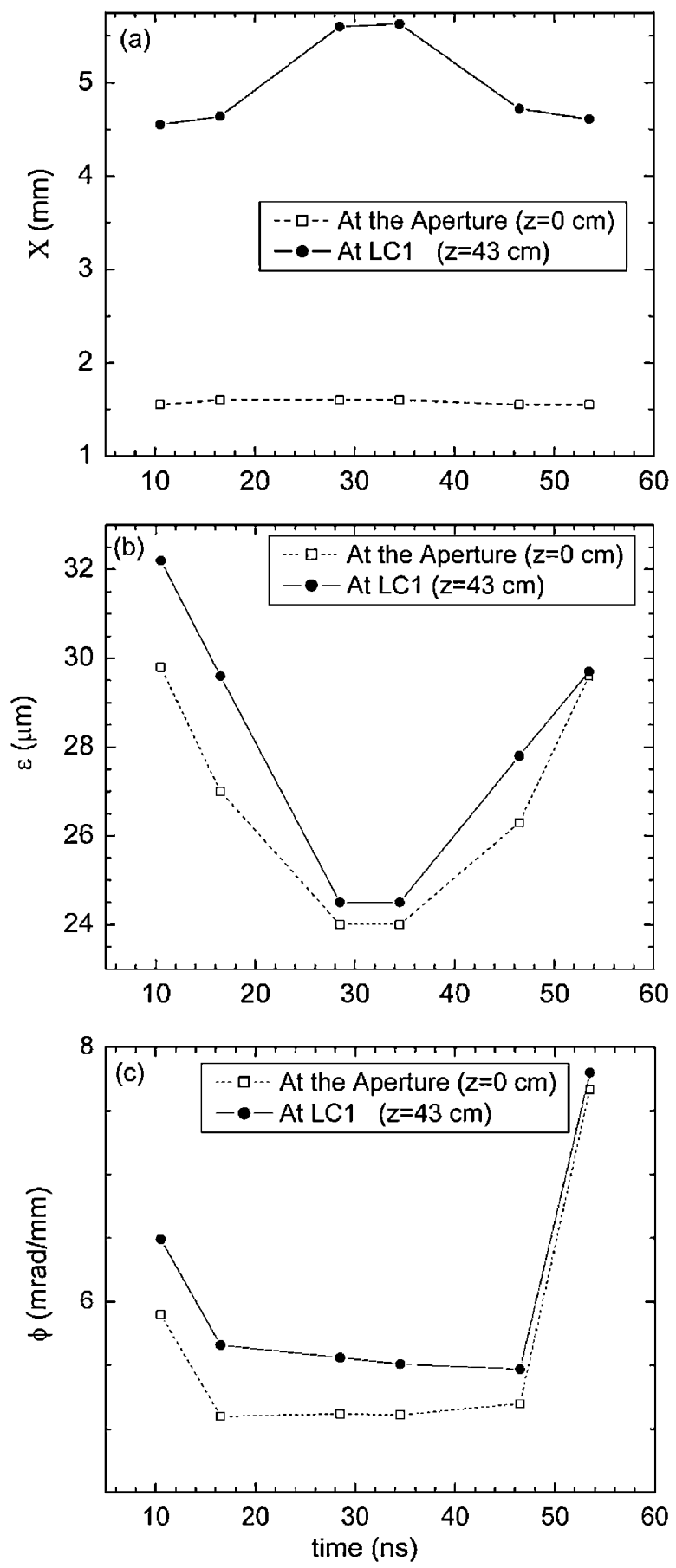

FIG. 8. Beam parameters measured in the experiment as a function of time along the pulse, at the screen location: (a) Beam size; (b) emittance; (c) slope of the phase space [from Eq. (10)]. The solid line corresponds to measurements at LC1 $(z=43.0 \mathrm{~cm})$ and the dashed line at the aperture $(z=$ $0 \mathrm{~cm})$.

the emittances are higher at the edges with respect to the center at both locations.

Another interesting observation is that not all the slices have the same orientation. This becomes apparent when we use Eq. (10) to calculate the slope of the phase space
[Fig. 8(c)]. Again the dashed line indicates the measured slope at the aperture. From Fig. 8(c) we can deduce some interesting physical insights: First, the slope is positive for both locations, suggesting that the beam is expanding. Second, like the emittance, the slope is varying rapidly near the beam ends. There may be more than one cause for this behavior. Note especially the rapid variation of the beam current within the edge slices a and $\mathrm{f}$ in Fig. 6. Then, as in the case of the perturbed rectangular beam, the phasespace orientation can change within the image integration gate, resulting in an apparent enlargement of the distribution in phase space. Furthermore, the tomography analysis assumes a constant, "average," current within each slice, which is clearly not the case in the ends. One solution for this problem would be to decrease the camera gate window so that the variation in current within each slice is reduced. Unfortunately, our present camera system is limited to a minimum 3 ns gate.

\section{CONCLUSION}

In summary, in this paper we reported on a proof-ofprinciple experiment for demonstrating the possibility of reconstructing the time-resolved phase-space distribution by tomography. Our results indicate that the transverse distribution is affected by the longitudinal density perturbations. We measured the detailed phase-space distribution in high resolution and were able to determine the beam initial conditions, such as the beam emittance, size, and phase-space slope. We believe that knowledge of those initial conditions in simulation codes can help to more accurately predict the evolution of the beam. Using a parabolic pulse we showed that the emittance is greater along the beam ends than near the center. Unfortunately, the camera gate is limited to $3 \mathrm{~ns}$ and, hence, it is unclear whether this effect is an artifact due to the rapid variation of current within those slices. However, our measurements are consistent with that at the exit of the SPARC photoinjector [20] where similar trends were observed for the transverse phase space and emittance.

It should be noted that this tomography diagnostic is not restricted to fluorescent screens. Hence, an interesting thought is the possibility to generalize the tomography technique described in this paper to high energy (5$100 \mathrm{MeV}$ range), short-pulse (sub-ps range) electron beams such as needed in the injectors for short-pulse $x$-ray FELs, for example, the Linac Coherent Light Source [33]. It may be possible to apply slice tomography to images collected by OTR [34], which has a sub-ps response, in combination with a faster gated ICCD or using a streak camera, if the beam is reproducible from shot to shot. In the latter case, information about the 2D beam distribution that is needed for tomography can be obtained by performing scans along different directions across the beam by suitably rotating or moving the input slit [35]. 


\section{ACKNOWLEDGMENTS}

We wish to acknowledge M. Walter, S. Bernal, S. M. Lund, D. Sutter, B. Beaudoin, D. Feldman, H. Li, V. Yakimenko, and C. Papadopoulos for helpful discussions. This work is supported by the U.S. Department of Energy High Energy Physics and Fusion Energy Science, and by the Department of Defense Office of Naval Research and Joint Technology Office.

[1] C. A. Brau, Free-Electron Lasers (Academic Press, San Diego, 1990).

[2] R. B. Palmer et al., in Proceedings of the 2007 Particle Accelerator Conference, Albuquerque, NM (IEEE, New York, 2007), p. 3193.

[3] G. H. Hoffstaetter et al., in Proceedings of the 2003 Particle Accelerator Conference, Portland, OR (IEEE, Piscataway, NJ, 2003), p. 192.

[4] J. Wei, Y. Y. Lee, D. Raparia, J. Sandberg, J. Tuozzolo, and W. T. Weng, in Proceedings of the 2003 Particle Accelerator Conference, Portland, OR, Ref. [2], p. 571.

[5] D. Dowell et al., Phys. Plasmas 4, 3369 (1997).

[6] K. Tian, Y. Zou, Y. Cui, I. Haber, R. A. Kishek, M. Reiser, and P. G. O’Shea, Phys. Rev. ST Accel. Beams 9, 014201 (2006).

[7] O. J. Luiten, S. B. van der Geer, M.J. de Loos, F. B. Kiewiet, and M.J. van der Wiel, Phys. Rev. Lett. 93, 094802 (2004).

[8] J.B. Rosenzweig, in Proceedings of the 2008 Advanced Accelerator Concepts Workshop, Santa Cruz, CA, 2008, http://aac08.lbl.gov/AAC08-WG6-orals_July26.pdf.

[9] T. Shaftan and Z. Huang, Phys. Rev. ST Accel. Beams 7, 080702 (2004).

[10] K. Bane et al., in Proceedings of the 2007 Particle Accelerator Conference, Albuquerque, NM (Ref. [2]), p. 807.

[11] H. H. Braun, R. Corsini, L. Groening, F. Zhou, A. Kabel, T. O. Raubenheimer, R. Li, and T. Limberg, Phys. Rev. ST Accel. Beams 3, 124402 (2000).

[12] L. Serafini, AIP Conf. Proc. 413, 321 (1997).

[13] P. Musumeci, J. T. Moody, R. J. England, J. B. Rosenzweig, and T. Tran, Phys. Rev. Lett. 100, 244801 (2008).

[14] D. Neuffer, IEEE Trans. Nucl. Sci. 26, 3031 (1979).
[15] F. M. Bieniosek et al., Nucl. Instrum. Methods Phys. Res., Sect. A 544, 481 (2005).

[16] J. R. Harris, R. B Feldman, and P. G. O'Shea, in Proceedings of the 2007 Particle Accelerator Conference, Albuquerque, NM (Ref. [2]), p. 3597.

[17] D. H. Dowell et al., Nucl. Instrum. Methods Phys. Res., Sect. A 507, 327 (2003).

[18] M. Rohrs et al., in Proceedings of the 10th European Particle Accelerator Conference, Edinburgh, Scotland, 2006 (EPS-AG, Edinburgh, Scotland, 2006), p. 77.

[19] X. Qiu et al., Phys. Rev. Lett. 76, 3723 (1996).

[20] D. Alesini et al., Nucl. Instrum. Methods Phys. Res., Sect. A 568, 488 (2006).

[21] K. Tian et al., Phys. Plasmas 15, 056707 (2008); K. Tian, Ph.D. dissertation, University of Maryland, College Park, 2008, Chap. 5.

[22] M. Reiser, Theory and Design of Charge Particle Beams (John Wiley \& Sons, Inc., New York, 1994); 2nd edition, published by Wiley-VCH in March 2008.

[23] B. E. Carlsten, Nucl. Instrum. Methods Phys. Res., Sect. A 285, 313 (1989).

[24] L. Serafini and J. B. Rosenzweig, Phys. Rev. E 55, 7565 (1997).

[25] C. B. McKee, P. G. O'Shea, and J. M. J. Madey, Nucl. Instrum. Methods Phys. Res., Sect. A 358, 264 (1995).

[26] V. Yakimenko, M. Babzien, I. Ben-Zvi, R. Malone, and X.-J. Wang, Phys. Rev. ST Accel. Beams 6, 122801 (2003).

[27] F. Zhou, A. Kabel, J. Rosenzweig, R. Agustsson, G. Andonian, D. Cline, A. Murokh, and V. Yakimenko, Phys. Rev. ST Accel. Beams 9, 114201 (2006).

[28] D. Stratakis, R. A. Kishek, H. Li, S. Bernal, M. Walter, B. Quinn, M. Reiser, and P. G. O'Shea, Phys. Rev. ST Accel. Beams 9, 112801 (2006).

[29] J. Radon, Math. Phys. Klasse 69, 262 (1917).

[30] A. C. Kak and M. Slaney, Principles of Computerized Tomographic Imaging (IEEE Press, New York, 1988).

[31] D. Stratakis, K. Tian, R. A. Kishek, I. Haber, M. Reiser, and P. G. O'Shea, Phys. Plasmas 14, 120703 (2007).

[32] J. G. Wang et al., IEEE Trans. Electron Devices 37, 2622 (1990).

[33] R. Akre et al., Phys. Rev. ST Accel. Beams 11, 030703 (2008).

[34] R. B. Fiorito and D. W. Dule, AIP Conf. Proc. 319, 21 (1994).

[35] S. Gierman, Ph.D. dissertation, University of California, San Diego, 1999. 